\title{
Deformation relief in crystals as a way of stress relaxation
}

\author{
E. A. Alfyorova ${ }^{1, \dagger}$, D. V. Lychagin ${ }^{1,2}$ \\ ${ }^{\dagger}$ katerina525@mail.ru
}

${ }^{1}$ Tomsk Polytechnic University, 30 Lenin avenue, 634050, Tomsk, Russia

${ }^{2}$ Tomsk State University, 36 Lenin avenue, 634050, Tomsk, Russia

The formation of a deformation relief of crystals is considered as a way of relaxation of an applied stress. The process of selforganization of slip bands into structural elements of the deformation relief of a qualitatively new level (packs of slip bands, meso- and macrobands) is directed to reduce local stresses in the zone of their formation as compared to those in the region occupied by slip bands. It is established that the formation of a quasiperiodic deformation relief occurs under the conditions of Azaro-Tiller-Greenfield instability and critical wavelengths of the surface perturbations are calculated. Each type of structural elements of the deformation relief causes a certain local curvature of the surface and increases the magnitude of the local stress in the region of its formation. On an example of nickel monocrystals it is revealed that the maximum stress concentration on the surface of 8.2 times is caused by packed slip bands, macrobands cause a 6.3 times increase of the local stress and the minimum concentration value of 3.3 is typical for mesobands. Curvature radii of the convex zones are the largest for macrobands $(r=0.40 \pm 0.04 \mu \mathrm{m})$ that facilitates the stress relaxation, while for mesobands it is the smallest $(\mathrm{r}=0.050 \pm 0.003 \mu \mathrm{m})$. It is shown that the process of self-organization of the deformation relief increases the size of a region with compatible strains that results in a longer preservation of the crystal integrity during deformation. On examples of f.c.c. single crystals of nickel, aluminum, ordered and disordered $\mathrm{Ni}_{3} \mathrm{Fe}$ alloy it is shown that the self-organization of slip bands into packs reduces the local stress in the packs formation zone by 1.8 to 3.5 times in comparison with slip bands. The revealed regularities are characteristic for materials with different stacking fault energies, types of sliding dislocations, and crystallographic features.

Keywords: deformation relief, f.c.c. single crystals and alloys, stress relaxation, confocal microscopy, surface curvature, local stress.

\section{Деформационный рельеф в кристаллах как способ релаксации напряжений}

\author{
Алфёрова Е.А. ${ }^{1, \dagger}$, Лычагин Д. В. ${ }^{1,2}$ \\ ${ }^{1}$ Национальный исследовательский Томский политехнический университет, пр. Ленина 30, 634050, Томск, Россия \\ ${ }^{2}$ Национальный исследовательский Томский государственный университет, пр. Ленина 36, 634050, Томск, Россия
}

В настоящей работе формирование деформационного рельефа кристалла рассматривается как способ релаксации приложенного напряжения. Показано, что процесс самоорганизации следов сдвига в структурные элементы деформационного рельефа качественно нового уровня (пачки следов, мезо- и макрополосы) направлен на снижение локальных напряжений в зоне их формирования по сравнению с областью, занятой следами сдвига. Установлено, что формирование квазипериодического деформационного рельефа происходит в условиях нестабильности Азаро-Тиллера-Гринфельда, рассчитаны критические длины волны возмущений поверхности $\lambda_{c}$ для пачек следов, мезо- и макрополос. Показано, что каждый тип структурных элементов деформационного рельефа вызывает определенную локальную кривизну поверхности и повышает величину локального напряжения в месте своего формирования. На примере монокристаллов никеля выявлено, что максимальное повышение напряжения в 8.2 раза в месте выхода на поверхности создают следы сдвига, макрополосы повышают локальное напряжение в 6.3 раз, а минимальное значение характерно для мезополос (в 3.3 раза). Радиус кривизны в зоне выпуклости для макрополос самый большой $(\mathrm{r}=0.40 \pm 0.04 \mu \mathrm{m})$, что способствует релаксации напряжений, а для мезополос самый маленький $(\mathrm{r}=0.050 \pm 0.003 \mu \mathrm{m})$. Показано, что процесс самоорганизации деформационного рельефа способствует увеличению размера области со взаимосогласованной деформацией, что способствует более длительному сохранению целостности кристалла при пластической деформации. На примере ГЦК-монокристаллов никеля, алюминия, а также упорядоченного и неупорядоченного сплава $\mathrm{Ni}_{3} \mathrm{Fe}$ показано, что самоорганизация следов сдвига в пачки снижает локальное напряжение в зоне формирования пачки в 1.8 - 3.5 раза по сравнению со следами сдвига. Установлена общность выявленных закономерностей для материалов с разными значениями ЭДУ, типом скользящей дислокации (степени порядка) и кристаллографическими особенностями.

Ключевые слова: деформационный рельеф, ГЦК-монокристаллы и сплавы, релаксация напряжений, конфокальная микроскопия, кривизна поверхности, локальное напряжение. 


\section{1. Введение}

Любое внешнее нагружение кристаллического твердого тела отражается в его формоизменении и формировании деформационного рельефа. Индивидуальные особенности этих процессов связаны с физико-механическими свойствами тех или иных металлов.

Общепризнанным механизмом деформации является механизм, связанный с трансляцией дефектов кристаллической решетки (дислокаций, дисклинаций, вакансий). Наиболее очевидным структурным элементом деформационного рельефа (СЭДР), образованным этим механизмом является след скольжения или след сдвига, которые мы можем наблюдать в оптический микроскоп (всегда при начальных степенях деформации). След сдвига состоит из пачки следов скольжения. Однако, во многих работах описываются картины деформационного рельефа, образованные не только следами сдвига, но и более сложными структурами [1-3]. Такими как пачки следов, мезополосы, макрополосы, гофры (складчатые структуры), которые в общем случае мы предлагаем называть структурными элементами деформационного рельефа. Следовательно, в процессе пластической деформации мы можем наблюдать самопроизвольное образование упорядоченных пространственных структур качественно иного уровня в сильно неравновесных открытых системах при приложении внешнего поля. Соответственно, можно говорить о самоорганизации сдвиговых процессов при пластической деформации, которая отражается в формировании деформационного рельефа различного типа. В этом случае рельеф можно рассматривать как диссипативную систему, благодаря которой рассеивается энергия, поступающая извне.

Очевидно, что целесообразность формирования определенного типа деформационного рельефа должна быть обусловлена обеспечением целостности кристалла при деформации (живучестью системы) при внешнем энергетическом воздействии. Отсюда логично вытекает вопрос какой тип структурных элементов деформационного рельефа является наиболее благоприятным для сохранения целостности кристалла при пластической деформации и почему следы сдвига самоорганизуются в СЭДР качественного другого уровня.

На сегодняшний день имеется большое количество работ, посвященное деформационному рельефу на поверхности поли- и монокристаллов [4-15]. Во многих работах по деформационному рельефу судят о неоднородности деформаций $[9,13-15]$. При этом могут использоваться как непосредственно характеристики рельефа (расстояние между следами сдвига, величина ступеньки и т. д.) [9], так и применяться дополнительные методы (метод корреляции цифровых изображений [14], метод делительных сеток [16] и др.), которые по изменению рельефа идентифицируют места неоднородного протекания деформации. Однако ответа на вопрос почему на поверхности формируются те или иные структурные элементы деформационного рельефа по-прежнему нет. Вероятно, виной тому отсутствие надежной информации о механизмах формирования деформационного рельефа и его роли в процессе самоорганизации пластической деформации.

Целью настоящей работы является определение роли структурных элементов деформационного рельефа различного типа в сохранении целостности кристалла при деформации.

\section{2. Материал и методика}

В настоящей работе описываются результаты, полученные на монокристаллах чистых металлов никеля, алюминия и сплава $\mathrm{Ni}_{3} \mathrm{Fe}$ с ближним (БП) и дальним (ДП) порядком. Использовали образцы размерами $3 \times 3 \times 6$ мм с ориентацией осей сжатия в углах стандартного стереографического треугольника. Деформация этих материалов при комнатной температуре протекает по октаэдрическим плоскостям скольжения, что делает их удобными материалами для исследования деформационного рельефа, формирующегося на поверхности кристалла после деформации скольжением. Однако, здесь следует отметить важную особенность упорядочивающихся сплавов. Пластическая деформация в них связывается с движением сверхдислокаций. Образцы имели форму тетрагональной призмы с соотношением высоты к ширине равной двум. Ориентировку осуществляли на рентгеновском аппарате ИРИС 3 по эпиграммам с точностью $\pm 1^{\circ}$, с уточнением ориентации на рентгеновском дифрактометре ДРОН-3, с точностью $\pm 0.02^{\circ}$. Поверхность образцов готовили механическим шлифованием и полированием и заключительной электролитической полировкой.

Деформирование сжатием проводили на испытательной машине Instron ElektroPuls E10000 со скоростью $1.4 \cdot 10^{-3} \mathrm{~s}^{-1}$ при комнатной температуре. Картину деформационного рельефа исследовали на оптическом микроскопе Leica DM 2500Р и конфокальном лазерном сканирующем микроскопе Olympus LEXT OLS4100.

\section{3. Экспериментальные результаты и обсуждения}

В настоящей работе рассматривались такие структурные элементы деформационного рельефа как следы сдвига, пачки следов сдвига, мезо- и макрополосы. Гофрированные деформационные структуры (складки) остались за рамками настоящей публикации, т. к. механизм их формирования связан не только с дислокационным скольжением, но и с разориентацией локальных областей в объеме кристалла [3]. Кроме того, при деформации ГЦК-монокристаллов они имеют большое морфологическое разнообразие $[2,17,18]$, что требует отдельной публикации.

Анализ деформационного рельефа может проводиться с различных точек зрения. При рассмотрении деформационного рельефа как способа сохранения сплошности кристалла при деформации интересным является подход на основе которого формирование рельефа рассматривается как способ рассеивания прилагаемого напряжения. В работе [19] представлено, что если поверхность твердого тела плоская, то его упругая деформация 
однородна. В этом случае и приложенное напряжение и вызванная им деформация будут иметь один и тот же знак, т. е. $\sigma_{x x} \neq 0, \sigma_{y y}=\sigma_{x y}=0$. Если же поверхность твердого тела не плоская, то поле напряжений в твердом теле становится неоднородным, т.е. $\sigma_{x x} \neq \sigma_{y y} \neq \sigma_{x y} \neq 0$. В этом случае, релаксация напряжений происходит на выступах, в менее стесненных условиях, тогда как во впадинах образуются концентраторы напряжений, которые геометрически более ограничены по сравнению с выступами. Результирующий градиент напряжений оказывает содействие более интенсивному формированию впадин, а это еще более усиливает градиент напряжений, что требует дальнейшей релаксации. Таким образом, благодаря развитию деформационного рельефа достигается баланс [19].

Анализ деформационного рельефа позволяет предположить, что в условиях сжатия кристаллов возрастает роль деформационного рельефа на свободных поверхностях кристалла и немаловажным становится вклад поверхностного механизма релаксации упругой энергии. Этот эффект известен как нестабильность Гринфельда [20]. Он был обнаружен независимо друг от друга несколькими учеными, поэтому его часто называют нестабильностью Азаро-Тиллера-Гринфельда [19-21]. Нестабильность Азаро-Тиллера-Гринфельда имеет упругое происхождение и протекает при относительно низких величинах упругих деформаций. Однако, на сегодняшний день имеются данные о том, что этот процесс характерен и для случаев, когда величина напряжений достаточна для осуществления пластической деформации [22-25]. Суть явления заключается в том, что кристалл имеет возможность снизить упругую энергию путем образования поверхностных модуляций, т.е. за счет формирования деформационного рельефа. При этом длина волны $\lambda$ определяется равновесием между упругой энергией, которая приводит к огрублению рельефа и поверхностным натяжением, которое стремится к сглаживанию поверхности. Критическая длина волны возмущений поверхности $\left(\lambda_{c}\right)$ в рамках линейного приближения может быть определена как [19,20]

$$
\lambda>\lambda_{c}=\pi \gamma_{g} E / \sigma^{2} \cdot\left(1-v^{2}\right)
$$

где $\gamma_{g}, E, \sigma$ и $v$ соответственно поверхностное натяжение, модуль Юнга, напряжение и коэффициент Пуассона, $\lambda$ - наблюдаемая в эксперименте длина волны. Величина поверхностного натяжения и напряжение могут быть определены из эксперимента. Выполнение этого соотношения позволит говорить о формировании деформационного рельефа в условиях нестабильности АзароТиллера-Гринфельда. В случае если $\lambda<\lambda_{c}$, поверхность сглаживается [19,26].

Величина поверхностного натяжения для $\gamma_{g}$ определялась из реального деформационного профиля поверхности путем анализа морфологии поверхности по методике изложенной в [27].

$$
\gamma_{g}=2 \sin \beta \gamma_{s}
$$

где $\gamma_{s}$ - поверхностная энергия на единицу площади (справочная величина), $\beta$ - угол наклона выступа элемента рельефа к плоскости кристалла (определялся из анализа экспериментальных данных).

Результаты расчета критической длины волны $\lambda$ и значения длины $\lambda$ из эксперимента для деформационных структур различного типа показаны в табл. 1 (в процентах указана степень деформации образца).

Таким образом, видно, что в рассматриваемом в работе случае период волны превышает критическую длину волны возмущений поверхности, т. е. соотношение (1) выполняется и происходящие процессы можно рассматривать с точки зрения нестабильности Азаро-ТиллераГринфельда.

Данные о морфологии деформационного рельефа, полученные с помощью конфокальной микроскопии, показывают, что каждый тип СЭДР вызывает определенную локальную кривизну поверхности на различных масштабных уровнях. Квазипериодический профиль представляет собой чередование выступов и впадин на макро- и мезоуровне (указано стрелками на рис. 1). На рис. 1 показан профиль поверхности вдоль секущей 1-1, ось $Y$ направлена перпендикулярно поверхности кристалла.

Табл. 1. Расчетные и экспериментальные длины волн.

Table 1. Calculated and experimental wavelengths.

\begin{tabular}{|c|c|c|}
\hline $\begin{array}{c}\text { СЭДР, степень деформации } \\
\text { Structural element of deformation } \\
\text { relief (SEDR), degree of strain }\end{array}$ & $\lambda_{c}, \mu \mathrm{m}$ & $\lambda, \mu \mathrm{m}$ \\
\hline $\begin{array}{c}\text { Пачки следов сдвига, 20\% } \\
\text { Рack of slip bands, 20\% }\end{array}$ & 72 & $101 \pm 9$ \\
\hline $\begin{array}{c}\text { Мезополосы, 22\% } \\
\text { Меsoband, 22\% }\end{array}$ & 92 & $99 \pm 19$ \\
\hline $\begin{array}{c}\text { Макрополосы, 31\% } \\
\text { Масroband, 31\% }\end{array}$ & 11 & $52 \pm 5$ \\
\hline
\end{tabular}
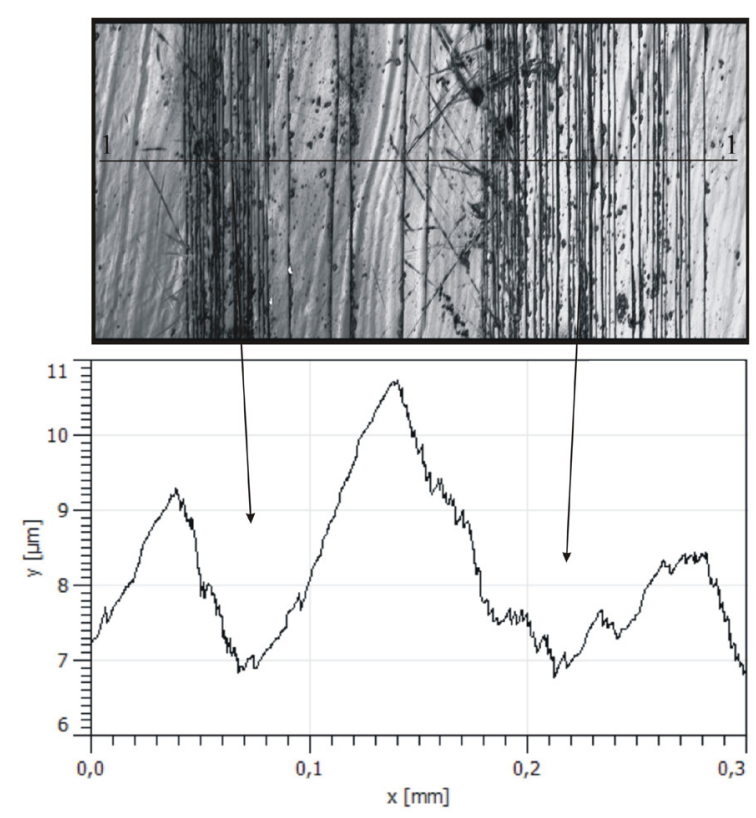

Рис. 1. Квазипериодический профиль поверхности (пачки следов сдвига указаны стрелками).

Fig. 1. The quasiperiodic surface profile (pack of slip bands are indicated by arrows). 
C позиции нестабильности Азаро-Тиллера-Гринфельда выступы являются местами релаксации напряжений, в то время как впадины - это концентраторы напряжений $[19-21,26]$. По сути впадины на поверхности представляют собой локальные зоны отрицательной кривизны поверхности, в которых возникают дополнительные сжимающие напряжения $\sigma_{a}$. Они в свою очередь приводят к росту локального напряжения. Приближенную оценку дополнительного сжимающего напряжения $\sigma_{a}$ можно провести используя соотношение механики

$$
\sigma_{a}=\sigma_{0} \cdot w
$$

где $w=\left(1+2 \cdot(\mathrm{a} / \mathrm{r})^{-1 / 2}\right), a-$ глубина впадины профиля, $r$ - радиус кривизны.

Нетрудно заметить, что коэффициент $w$ в формуле (3) фактически оценивает степень повышения локального напряжения тем или иным типом СЭДР.

Расчеты на примере монокристаллов никеля, показали, что разница в степени повышения локального напряжения составляет порядка $40 \%$. При этом максимальное повышение напряжения дают макрополосы деформации $w=6.3$, а минимальное - мезополосы $w=3.3$. При этом радиус кривизны в зоне выпуклости для макрополос самый большой $(\mathrm{r}=0.4 \pm 0.04 \mu \mathrm{m})$, что способствует релаксации напряжений, а для мезополос самый маленький $(\mathrm{r}=0.05 \pm 0.003 \mu \mathrm{m})$. Следы сдвига до самоорганизации их в СЭДР повышают локальное напряжение в 8.2 раза, а учитывая, что радиус кривизны в зоне выпуклости для следов сдвига менее $0.01 \mu \mathrm{m}$, то процесс релаксации напряжений возможен только при начальной степени деформации. С использованием данной методики была оценена величина дополнительного напряжения на границе между твидовой структурой и продольными макроскопическими полосами в работе [22]. Было определено, что формированием макрополос приводит к повышению локального сжимающего напряжения.

Аналогичные расчеты были проведение для ГЦКметаллов и сплавов с различным значением энергии дефекта упаковки (ЭДУ). Результаты приведены в табл. 2. В данном случае рассматривался процесс самоорганизации следов сдвига в пачки. Он наиболее характерен для [001]-монокристаллов с кубическими боковыми гранями [7,28,29].

Анализируя полученные результаты видим, что процесс развития пачек следов сдвига способствует снижению локального напряжения $\sigma=\sigma_{0}+\sigma_{a}$ на мезо- и макроуровне по сравнению с отдельными следами сдвига, а, следовательно, и сохранению целостности кристалла при деформации. Самоорганизация следов в пачки сни- жает локальное напряжение в зоне формирования пачки в 1.8 - 3.5 раза по сравнению со следами сдвига.

Построение автокорреляционной функции для каждого типа СЭДР позволило определить радиус корреляции $r_{k}$, по которому можно оценить размер области с самосогласованной деформацией. Радиусы корреляции для СЭДР различного типа для никеля составляют: для следов сдвига $37 \pm 9 \mu \mathrm{m}$, для мезополос $-42 \pm 8 \mu \mathrm{m}$, для макрополос - $43 \pm 5 \mu \mathrm{m}$. Результаты, полученные для других металлов и сплавов приведены в табл. 2 (в процентах указана степень деформации образца). При самоорганизации следов в пачки можно наблюдать увеличение размера области с самосогласованной деформацией.

Таким образом, самоорганизация сдвиговых процессов при деформации, которая отражается в формировании СЭДР различного типа приводит к увеличению области с взаимосогласованной деформацией. Это способствует сохранению целостности кристалла при нагружении и однородному протеканию деформации.

\section{4. Выводы}

Показано, что формирование деформационного рельефа происходит в условиях нестабильности Азаро-Тиллера-Гринфельда. Образование структурных элементов деформационного рельефа различного типа происходит с целью диссипации приложенного напряжения.

Процесс самоорганизации сдвиговых процессов при пластической деформации из следов сдвига, которые формируются при начальных степенях деформации в СЭДР качественно нового уровня (пачки следов, мезои макрополосы) способствует снижению локальных напряжений на мезо- и макроуровне в зоне формирования этих СЭДР по сравнению с областью занятой следами сдвига.

Процесс самоорганизации сдвиговых процессов, выражающийся в формировании деформационного рельефа, способствует увеличению размера области со взаимосогласованной деформацией, что способствует более длительному сохранению целостности кристалла при пластической деформации.

Указанные выше процессы и закономерности присущи различным материалам с учетом ЭДУ, кристаллографических особенностей и типа скользящей дислокации (степени порядка).

В заключении авторы выражают благодарность к.т.н., м.н.с. ИФПМ СО РАН А.В. Филиппову за помощь в получении экспериментальных результатов.

Табл. 2. Значения коэффициента $w$ и радиуса корреляции $r_{k}$.

Table 2. Coefficient $w$ values and correlation radius $r_{k}$.

\begin{tabular}{|c|c|c|c|c|c|}
\hline $\begin{array}{c}\text { Тип СЭДР } \\
\text { Type of SEDR }\end{array}$ & $\begin{array}{l}\text { Параметр } \\
\text { Parameter }\end{array}$ & $\begin{array}{c}\mathrm{Ni}_{3} \mathrm{Fe} \text { БП, } 20 \% \\
\mathrm{Ni}_{3} \text { Fe short-range order, } 20 \%\end{array}$ & $\begin{array}{c}\mathrm{Ni}_{3} \mathrm{Fe} \text { ДП, } 16 \% \\
\mathrm{Ni}_{3} \text { Fe long-range order, 16\% }\end{array}$ & $\mathrm{Al}, 27 \%$ & $\mathrm{Ni}, 20 \%$ \\
\hline \multirow{2}{*}{$\begin{array}{l}\text { Следы сдвига } \\
\text { Slip bands }\end{array}$} & $w$ & 13.1 & нет данных & 7.6 & 8.2 \\
\hline & $r_{k}, \mu \mathrm{m}$ & $15.7 \pm 0.9$ & нет данных & $45.3 \pm 1.5$ & $21 \pm 1.3$ \\
\hline \multirow{2}{*}{$\begin{array}{c}\text { Пачки следов сдвига } \\
\text { Pack of slip bands }\end{array}$} & $w$ & 3.8 & 2.3 & нет данных & 4.7 \\
\hline & $r_{k}, \mu \mathrm{m}$ & $33.5 \pm 0.6$ & $14.8 \pm 1.1$ & нет данных & $32 \pm 0.4$ \\
\hline
\end{tabular}


Благодарность/Aknowledgements. Исследование выполнено при финансовой поддержке РФФИ в рамках научного проекта № 16-32-60007 мол_а_дк. The reported study was funded by RFBR, according to the research project No. 16-32-60007 mol_a_dk.

\section{Список литературы}

1. S. Khoddam, H. Beladi, P. D. Hodgson, A. Zarei-Hanzaki, Surface wrinkling of the twinning induced plasticity steel during the tensile and torsion tests // Mater. Des. Elsevier Ltd, 2014. Vol. 60. P. $146-152$.

2. D. V. Lychagin, E.A. Alfyorova Folding in FCC metal single crystals under compression // Phys. Solid State. 2015. Vol. 57, № 10. P. $2034-2038$.

3. D.V. Lychagin, S. Yu. Tarasov, A.V. Chumaevskii, E. A. Alfyorova Strain-induced folding on [111] copper single crystals under uniaxial compression // Appl. Surf. Sci. Elsevier B. V., 2016. Vol. 371. P. 547 - 561.

4. M. Cai, S. C. Langford, J. Thomas Dickinson Orientation dependence of slip band formation in single-crystal aluminum studied by photoelectron emission // Acta Mater. Acta Materialia Inc., 2008. Vol. 56, №20. P. $5938-5945$.

5. G. Girardin, C. Huvier, D. Delafosse, X. Feaugas Correlation between dislocation organization and slip bands: TEM and AFM investigations in hydrogencontaining nickel and nickel-chromium // Acta Mater. Acta Materialia Inc., 2015. Vol. 91. P. 141-151.

6. S. Ha, K. Kim Heterogeneous deformation of Al single crystal: Experiments and finite element analysis // Math. Mech. Solids. 2011. Vol. 16, № 6. P. 652 - 661.

7. D. E. Kramer, M. F. Savage, L. E. Levine AFM observations of slip band development in $\mathrm{Al}$ single crystals // Acta Mater. 2005. Vol. 53, № 17. P. 4655 - 4664.

8. Tatsuya Okada, Mitsuyoshi Utani, Atsushi Osue, Nobukazu Fujii Minoru Tagami and Fukuji Inoko Slip Morphology and Recrystallization in Copper Single Crystals Tensile-Deformed along (011) and (001) Direction // Mater. Trans. 2005. Vol. 46, № 3. P. 602-607.

9. L. A. Teplyakova, I. V. Bespalova, D. V. Lychagin Spatial organization of deformation in aluminum [112] single crystals in compression // Phys. Mesomech. 2009. Vol. 12. P. $166-174$.

10. H.S. Ho, M. Risbet, X. Feaugas, G. Moulin The effect of grain size on the localization of plastic deformation in shear bands // Scr. Mater. 2011. Vol. 65, № 11. P. 998 - 1001.

11. J. Man, P. Klapetek, O. Man, A. Weidner, K. Obrtlík, J. Polák Extrusions and intrusions in fatigued metals. Part 2. AFM and EBSD study of the early growth of extrusions and intrusions in 316L steel fatigued at room temperature // Philos. Mag. 2009. Vol. 89, № 16. P. $1337-1372$.

12. J. Man, M. Valtr, M. Petrenec, J. Dluhoš, I. Kubena, K. Obrtlík, J. Polák AFM and SEM-FEG study on fundamental mechanisms leading to fatigue crack initiation // Int. J. Fatigue. 2015. Vol. 76. P. $11-18$.

13. L. A. Teplyakova, E.V. Kozlov Formation of scale structural levels of plastic deformation localization in metal single crystals. I. Macrolevel // Phys. Mesomech. 2006. Vol. 9, № 1 - 2. P. $53-62$.
14. X. G. Wang, J. F. Witz, A.E. Bartali, A. Oudriss, R. Seghir, P. Dufrénoy, X. Feaugas, E. Charkaluk A dedicated DIC methodology for characterizing plastic deformation in single crystals // Exp. Mech. 2016. Vol. 56, №7. P. $1155-1167$.

15. K.R. Magida, J.N. Florandob, D.H. Lassilab, M. M. LeBlancb, N. Tamurac and J. W. Morris Jr. Mapping mesoscale heterogeneity in the plastic deformation of a copper single crystal // Philos. Mag. 2009. Vol. 89, № 1. $77-107 \mathrm{p}$.

16. Lychagin D. V., Alfyorova E. A., Starenchenko V. A. Effect of crystallogeometric states on the development of macrobands and deformation inhomogeneity in [111] nickel single crystals // Phys. Mesomech. 2011. Vol. 14, № $1-2$. P. $66-78$.

17. D. V. Lychagin, S.Yu. Tarasov, A.V. Chumaevskii, E. A. Alfyorova Macrosegmentation and strain hardening stages in copper single crystals under compression // Int. J. Plast. Elsevier Ltd, 2015. Vol. 69. P. 36 - 53.

18. D. Lychagin, A. Chumaevskiy, E. Alferova Laws of development of deformation folds in [111] copper single crystal at axis compression // Appl. Mech. Mater. 2014. Vol. 682. P. $448-452$.

19. D. J. Srolovitz On the stability of surfaces of stressed solids // Arta Met. 1989. Vol. 37, № 2. P. 621 - 625.

20. M. A. Grinfeld (1986). 1 // Sov. Phys. Dokl. 31,. 1986. Vol. 31. P. $831-834$.

21. R. Asaro, W. Tiller Interface morphology development during stress corrosion cracking: Part I. Via surface diffusion // Metall. Trans. 1972. Vol. 3, №7. P. $1789-1796$.

22. P. V. Kuznetsov, Yu.I. Tyurinb, I.P. Chernov, T. I. Sigfussonc Grinfeld instability as a mechanism of the formation of self-similar structures on aluminum singlecrystal foils under cyclic tension // Phys. Solid State. 2012. Vol. 54, № 12. P. 2429 - 2436.

23. P.V. Kuznetsov, V.E. Panin, I.V. Petrakova Grinfeld instability in the formation of a tweed structure at the $\mathrm{Al}$ crystal surface under cyclic tension // Phys. Mesomech. 2010. Vol. 13, № 1 - 2. P. 70 - 78.

24. W. H. Yang, D. J. Srolovitz Surface morphology evolution in stressed solids: surface diffusion controlled crack initiation // J. Mech. Phys. Solids. 1994. Vol. 42, № 10. P. 1551.

25. R. Spatschek, E.A. Brener Fracture and the Grinfeld instability // J. Cryst. Growth. 2005. Vol. 275, № 1-2. P. $307-311$.

26. D.H. Yeon, P.R. Cha, M. Grant Phase field model of stress-induced surface instabilities: Surface diffusion // Acta Mater. 2006. Vol. 54, № 6. P. $1623-1630$.

27. W.W. Mullins Theory of thermal grooving // J. Appl. Phys. 1957. Vol. 28, № 3. P. $333-339$.

28. L. A. Teplyakova, D. V. Lychagin, E.V. Kozlov Shear localization in deformed $\mathrm{Al}$ single crystals with a compression axis orientation [001] // Phys. Mesomech. 2003. Vol. 6, № 1-2. P. 19-24.

29. D. V. Lychagin, E. A. Alfyorova, A.S. Tailashev Misorientation Development During the Formation of Macrobands in the [001] Nickel Single Crystals // Russ. Phys. J. 2015. Vol. 58, № 5. P. 717-723. 